\title{
APRENDIZAJE EN SERVICIO EN EL PROGRAMA DE FORMACIÓN FUNDAMENTAL DE LA UNIVERSIDAD DE TALCA, CHILE
}

\section{LEARNING IN SERVICE IN THE FUNDAMENTAL TRAINING PROGRAM OF THE UNIVERSITY OF TALCA, CHILE}

\author{
GILMA ÁLAMO SÁNCHEZ*, PAULA MANRÍQUEZ NOVOA**
}

Rec.: 07-10-2019 Acept:: 20-05-2020 Publ:: 30-jun-2020

http://doi.org/10.29035/ucmaule.58.59

\section{RESUMEN}

Se desarrolla una investigación descriptiva documental que sistematiza la experiencia del enfoque de competencias de la Universidad de Talca en el Programa de Formación Fundamental, basado en la metodología de aprendizaje en servicio, centrada en el análisis de las metodologías participativas y requerimientos institucionales universitarios. Describe la metodología de aprendizaje en servicio, sustentado en autores como Tobón y Quezada, formación integral y competencias; Mendía, aprendizaje en servicio; Folgueiras y Sabariego, IAP. Esta metodología desarrolla competencias de saber, saber hacer y saber ser, aplicando conocimientos adquiridos durante su formación profesional en un proyecto de intervención social con características de responsabilidad social.

Palabras clave: formación Integral, aprendizaje en servicio, investigación-acción.

\begin{abstract}
A descriptive documentary research is developed that systematizes the experience of the competency approach of the University of Talca in the Fundamental Training Program, based on the service learning methodology, focused on the analysis of participatory methodologies and university institutional requirements. It describes the service-learning methodology, supported by authors such as Tobón and Quezada, comprehensive training and skills; Mendía, service learning; Folgueiras and Sabariego, IAP. This methodology develops skills of knowing, knowing how to do and knowing how to be, applying knowledge acquired during their professional training in a social intervention project with characteristics of social responsibility.
\end{abstract}

Key words: Integral Training, service learning, action research.

* Vicerrectoría de pregrado. Programa de Formación Fundamental. Universidad de Talca. galamo@utalca.cl

** Vicerrectoría de pregrado. Universidad de Talca. pmanriq@utalca.cl 


\section{INTRODUCCIÓN}

La educación como hecho social, al institucionalizarse en el Estado, se ha ido transformando y extendiendo a todos los sectores de la sociedad, ampliando, por decirlo de alguna manera, sus funciones socializadoras y posibilitando la introducción de valores, normas y costumbres propias de la cultura o sociedad en la que se inserta. Esto "ha permitido cierta homogeneización de los contenidos explícitos y, por tanto, de sus fines o funciones sociales" (Castillo 2012, p. 73). Visto así, la educación, por medio de las instituciones, persigue entre sus fines la reproducción de la estructura social a través de la formación ciudadana, posibilitando un ser humano crítico y reflexivo, capaz de enfrentar la resistencia social y participar de los procesos de cambio.

En este contexto la Universidad de Talca (UTALCA) atendiendo a su misión, visión y valores compartidos, entiende la importancia de formar profesionales con sólidas competencias disciplinares y un alto sentido de pertenencia y responsabilidad con su entorno, lo cual posibilita la incorporación de la responsabilidad social desde lo ético como elemento no sólo de la transformación curricular sino en la innovación de las estrategias de enseñanza-aprendizaje y propicia la apertura de nuevos escenarios de participación hacia los grupos de interés dentro de los diversos ámbitos del quehacer académico.

Así vemos cómo se armoniza la línea de acción de la institución con las necesidades de la sociedad en la que se inserta, teniendo en cuenta la generación y transferencia de conocimientos como elementos constituyentes y esenciales de la misión universitaria, siendo esto un aporte para el bien común con énfasis en la interdisciplinariedad, propiciando el equilibrio entre la investigación básica y la investigación aplicada.

En este sentido, el siguiente artículo se propone dar a conocer, en el contexto de la sociología educativa, una experiencia de aplicación de la metodología de aprendizaje en servicio durante el año 2018 en el Programa de Formación Fundamental (PFF) para los estudiantes de diferentes carreras, enmarcado en el modelo de educación por competencias propio de la misma universidad.

\section{Modelo de educación basado en competencias en la UTALCA}

Este modelo establece el desarrollo del pensamiento crítico mediante el cual el estudiante reconoce, define y resuelve diversas situaciones asociadas a su 
perfil profesional; recoge y analiza datos e interpreta resultados; lee, critica y evalúa material escrito y enfoca una situación o problema desde una perspectiva nueva, original o imaginativa. Apoyando esta concepción, para Tobón (2013) la formación de competencias

debe contextualizarse en el marco de una comunidad determinada para que posea pertinencia y pertenencia. $Y$ el reto del entorno comunitario es validar tal formación y promoverla, buscando que se refuerce y complemente con el apoyo de otras instituciones sociales tales como la familia, las redes de apoyo social, las actividades recreativas y deportivas, los escenarios culturales y los medios masivos de comunicación. Pues es imposible generar impacto en la formación de competencias de alto nivel si no hay acuerdo y coherencia entre las instituciones educativas y los procesos sociales que permean e influyen en las personas (p. 32).

Este mismo autor plantea cinco ejes en la formación de competencias donde señala que "la formación de competencias no es responsabilidad solamente de las instituciones educativas, sino también de la sociedad, del sector laboral-empresarial, de la familia y de la persona humana" (Ibíd.), siendo estos ejes:

Responsabilidad de las instituciones educativas: consiste en implementar procesos pedagógicos y didácticos de calidad, con recursos suficientes, autovaloración continua basada en estándares de calidad y talento humano capacitado para tal propósito (directivos y docentes).

Responsabilidad social: es la promoción de una cultura de formación del talento humano con idoneidad, fortaleciendo los valores de solidaridad y cooperación, incidiendo en los medios de comunicación y aportando los recursos económicos necesarios en este propósito.

Responsabilidad del sector laboral-empresarial-económico: consiste en participar activamente en la formación de competencias mediante su integración con el sistema educativo y social.

Responsabilidad de la familia: consiste en formar a sus miembros en valores de convivencia y respeto, así como en habilidades básicas de pensamiento. 
Responsabilidad personal: es la formación de las propias competencias desde la autogestión del proyecto ético de vida. (Tobón, 2013, p. 35)

En estos cinco ejes se expresa el modelo de competencias de la Universidad de Talca, enfatizando los distintos niveles de responsabilidad aquí descritos y que son parte de la formación integral del estudiante a lo largo de su formación profesional. Por otra parte, parafraseando a Quezada (2014), el modelo de currículo por competencias conlleva a que las instituciones de educación universitaria realicen grandes esfuerzos diseñando proyectos y enfrentando los desafíos que impone el contexto educativo nacional e internacional en su vinculación con el mercado laboral, para dar respuesta a la formación de personas mediante el perfeccionamiento de los instrumentos cognoscitivos, teóricos e instrumentales del quehacer de la docencia e investigación. Según este autor, el objetivo es encontrar estrategias pertinentes que los hagan competitivos ante las realidades complejas, emergentes y en constante transformación, lo que exige su aprendizaje, actualización e innovación permanente.

Bajo este modelo educativo la elaboración del currículo se sustenta en el rol que debe desempeñar el docente como guía del proceso de enseñanza en tres ámbitos básicos del perfil que se desea del estudiante, a saber: la dimensión ético-valórica, la cual considera los principios de ciudadanía y convivencia humana; la dimensión académica, que incluye los conocimientos sobre la disciplina y la capacidad para investigar y generar conocimiento; y la dimensión profesional, en la que aporta su experiencia como elemento para competir en condiciones de igualdad y, de ser posible, con mayores ventajas en el mercado laboral.

Por tanto, la educación, desde este punto de vista, debe cambiar las ideas preconcebidas con el objetivo de proporcionar al alumno las competencias relevantes para su desempeño por medio de recursos didácticos que le permitan el salto de lo pasivo a lo activo. Visto así, el modelo se entiende "como un saber actuar en un contexto particular [donde intervienen] recursos personales y contextuales (saber, saber hacer, saber ser) para la solución de un problema específico, con un proceso de reflexión sobre lo que se está haciendo" (Quezada, 2014, p. 8). Partiendo de estos planteamientos, la UTALCA en su Plan Estratégico 2015 establece que estas competencias se expresan en las siguientes líneas de Formación Curricular: 


\section{Formación Fundamental}

\section{Formación Básica y}

\section{Formación Disciplinar}

La línea de Formación Fundamental, a su vez, se operativiza en el Programa de Formación Fundamental (PFF) a través de las líneas académicas Comunicación Oral y Escrita, Habilidades Interpersonales y Responsabilidad Social, las cuales se expresan en trayectorias de aprendizaje donde se explica el recorrido que harán los estudiantes durante su formación profesional, y se compone por competencia, aprendizaje y saberes.

Estos criterios permiten que el estudiante, desde la línea Comunicación Oral y Escrita utilice elementos de contenido, organización y métodos apropiados en la comunicación escrita; haga presentaciones formales e informales; escuche atenta y efectivamente; organice la información en forma lógica para apoyar sus conclusiones y acceda y comunique información utilizando las tecnologías de información y comunicación a su alcance.

La línea Habilidades Interpersonales facilita la organización de actividades en función de una meta, donde el estudiante comprende la dinámica del trabajo grupal en la conformación de equipos de trabajo para la resolución de conflictos; organiza, asigna prioridades y delega tareas; valoriza las diferencias individuales de las personas en las organizaciones y comprende el proceso de toma de decisiones grupales para el logro de objetivos institucionales.

Finalmente, la línea de Responsabilidad Social desarrolla en el estudiante sensibilidad hacia el trabajo mediante la ejecución sistemática de acciones orientadas a solucionar problemas, capturando oportunidades desde su profesión. En este punto se aportan herramientas de aprendizaje complejo, como señala Tobón (2013) "complementa la epistemología sistémica posibilitando un método de construcción de saberes que tiene en cuenta el entretejido de las partes, la construcción de relaciones, el caos, el cambio y la incertidumbre" (p. 12), con vinculación transdiciplinaria para entender las situaciones que en la práctica se presentarán. Así mismo se refuerzan los conceptos de ética como filosofía y de moral como principios, normas y valores a ser demostrados en el futuro ejercicio profesional.

De esta manera, aporta a su desarrollo individual y da respuesta a las necesidades de formación integral con una visión global y socialmente responsable, aumentando las oportunidades de empleabilidad futura. 
Es decir, comprende las responsabilidades sociales del individuo y las organizaciones; los roles de sus valores personales y profesionales en la toma de decisiones; ejercita habilidades de razonamiento ético y moral en la selección de opciones; comprende limitaciones personalesy organizacionales, desarrollando conciencia sobre la diversidad de las problemáticas a las cuales se enfrenta.

En este contexto surge esta investigación considerando dos aspectos principales: la necesidad académica de desarrollar competencias en responsabilidad social y la enseñanza de metodologías participativas para que el estudiante adquiera habilidades y destrezas en este componente de su formación profesional. Estos aspectos a su vez se fundamentan en la relevancia de las metodologías participativas, el conocimiento de la intervención social, los cambios en el proceso de enseñanza-aprendizaje de manera práctica y participativa y los nuevos requerimientos laborales para los egresados de todas las carreras de la UTALCA.

Por otra parte, el Programa de Formación Fundamental de la Universidad de Talca, surge a partir del año 2006 comenzando como un ramo formativo que busca complementar los conocimientos adquiridos por los estudiantes en su formación con la práctica profesional bajo criterios de Responsabilidad Social. En sus inicios, el estudiante desarrollaba un trabajo escrito donde plasmaba sus conocimientos con respecto a una problemática social determinada. Posteriormente se estructura en un año donde se dictan clases de Ética y Responsabilidad Social ( $7^{\circ}$ semestre) aplicando estos conocimientos en una práctica de índole social en vinculación con socios comunitarios ( $8^{\circ}$ semestre).

Partiendo de este planteamiento se desarrolla una investigación cuyo objetivo es sistematizar y dar a conocer una experiencia de aplicación del enfoque de competencias de la Universidad de Talca en el Programa de Formación Fundamental, durante el año 2018 con énfasis en la metodología de aprendizaje en servicio. Este objetivo se centra en el análisis de los argumentos de lo expuesto con anterioridad, englobando los siguientes aspectos: tendencias educativas en el contexto de las metodologías participativas y requerimientos institucionales universitarios actuales.

\section{Metodología de aprendizaje en servicio (MAS)}

Se considera, a los propósitos de la presente investigación, como una secuencia de pasos a seguir para entender, interpretar y, en la medida de lo posible, modificar o transformar la realidad y mejorar el aprendizaje. Se 
inserta en el conjunto de actividades que llevan a cabo tanto los docentes como los estudiantes, y conecta con las propuestas innovadoras que se dan en la institución: educación basada en competencias, aprendizaje basado en proyectos o problemas, aprendizaje cooperativo y colaborativo, aprendizaje en servicio, aprender a emprender, convivencia positiva. Así vemos como el aprendizaje en servicio puede ser considerado como "una propuesta educativa que combina procesos de aprendizaje y de servicio a la comunidad en un solo proyecto bien articulado en el que los participantes se forman al trabajar sobre necesidades reales del entorno con el objetivo de mejorarlo" (Mendía, 2016, p. 4). Este autor señala tres características básicas a saber:

Protagonismo activo: Donde la actividad está protagonizada activamente por estudiantes con acompañamiento formal de docentes

Servicio solidario: El cual se presta con la finalidad de atender necesidades reales y sentidas de una comunidad. Se planifican actividades concretas, adecuadasy acotadas a la edad y capacidades de los protagonistas, y orientadas a colaborar en la solución de problemáticas comunitarias específicas.

Aprendizajes intencionadamente planificados: Se realizan con la actividad solidaria articulando el aprendizaje de contenidos curriculares.

Para la línea de Responsabilidad Social, la Metodología de Aprendizaje en Servicio (MAS) es entendida como la prestación de un servicio desarrollado por parte de los estudiantes, destinado a cubrir necesidades reales de una comunidad organizada, planificado institucionalmente en forma integrada con el currículo en función del propio aprendizaje y vinculado estrechamente con la formación profesional. La utilización de esta metodología genera beneficios ya que se estimula la formación de actitudes participativas y solidarias, permitiendo una temprana sensibilización hacia ciertas problemáticas sociales y ambientales, porque se ofrece un clima institucional abierto a las realidades sociales y, en algunos casos, se permite la posibilidad de aprender procedimientos básicos de gestión referidos a su carrera. Persigue entre sus propósitos que los estudiantes de las diferentes carreras de la UTALCA en el cuarto año de su carrera realicen un proyecto de servicio solidario, concientizando su actuación con sentido ético y responsabilidad social, considerando la innovación y el desarrollo sustentable para su futuro ejercicio profesional. En la siguiente figura se puede observar como la 
Metodología de Aprendizaje en Servicio se integra en el modelo de educación por competencias de la UTALCA.

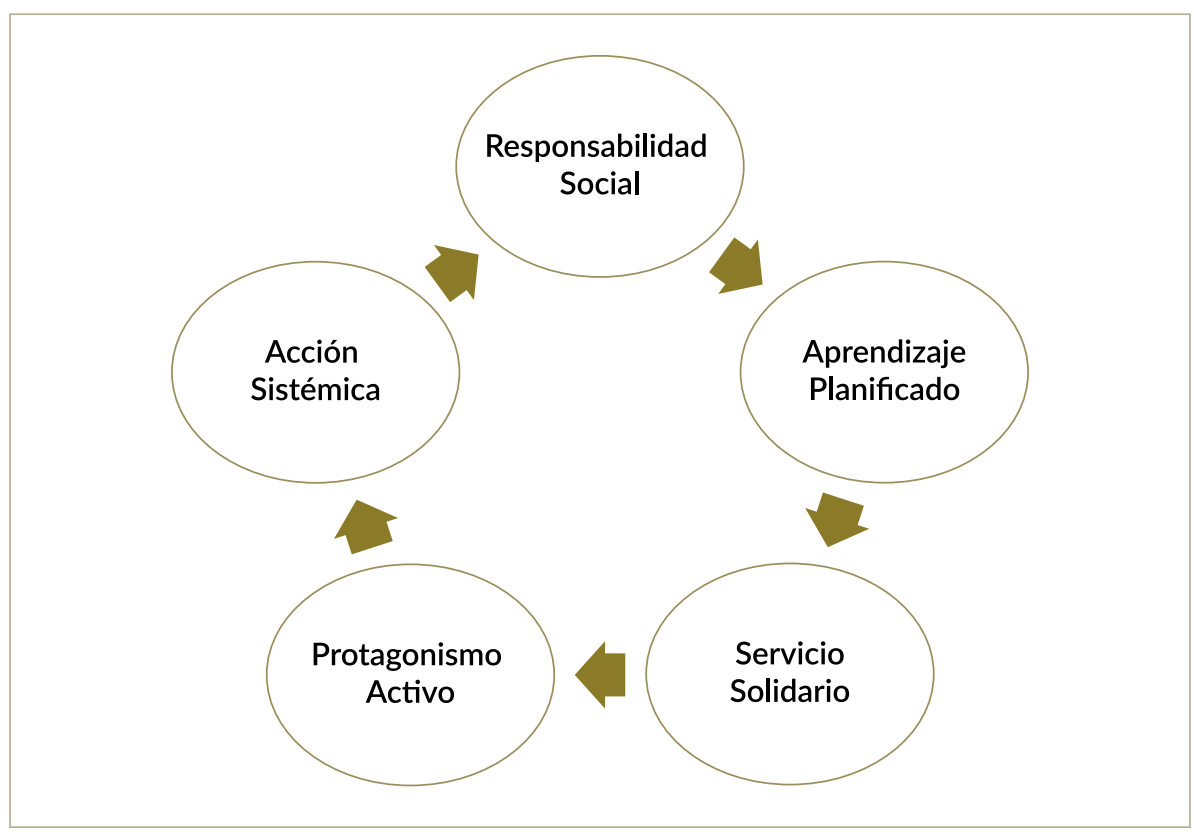

Figura 7. Aprendizaje en Servicio para el Programa de Formación Fundamental.

Para esta investigación se asume lo propuesto por Mendía (2016), ya que lo planteado por este autor se asemeja a la propuesta que a continuación se elabora con respecto al aprendizaje en servicio del estudiante universitario. Visto así, se combinan el aprendizaje y el servicio comunitario en un proyecto donde se plasman las diferentes etapas del proceso de intervención social en interacción con socios comunitarios para beneficio de los destinatarios; se asume el aprendizaje en servicio como parte de la formación fundamental del estudiante futuro egresado como profesional de la UTALCA. En este contexto, la metodología de aprendizaje en servicio facilita el acceso a las prácticas innovadoras apoyándose en la Investigación-acción participativa (IAP) como herramienta de investigación social.

Su aplicación establece que los estudiantes realicen actividades dirigidas a la atención de necesidades comunitarias reales en forma integrada con el currículo de su carrera (Universidad de Talca, 2018). De esta forma, el Programa de Formación Fundamental la utiliza en la línea Responsabilidad Social ya que 
los estudiantes en los semestres $7^{\circ}$ y $8^{\circ}$ de su respectiva formación académica comienzan, a nivel de terreno, con un acercamiento al socio comunitario beneficiario del proyecto de intervención social que realizará, sustentado en los conocimientos obtenidos a través de las clases donde se le facilita el desarrollo de competencias en responsabilidad social y se le brindan técnicas para la intervención social. Estas técnicas consisten en la elaboración y aplicación de instrumentos para realizar un diagnóstico participativo de forma tal que se oriente la actuación a las áreas susceptibles de ser intervenidas. Esto permite al estudiante poner en práctica sus conocimientos en total sincronización con el socio comunitario respectivo y con los beneficiarios del proyecto, de acuerdo con las ramas profesionales que se deben abordar. En el séptimo semestre se desarrollan, académicamente, los conceptos para la intervención social sustentados en Ética y Responsabilidad Social, los cuales fortalecen sus competencias para la elaboración de un proyecto social. En el octavo semestre se propicia la intervención con los socios comunitarios siguiendo las etapas de Contacto inicial, donde se conocen y reconocen las necesidades a satisfacer con la intervención propiamente dicha a través del desarrollo del proyecto socio comunitario; se realiza el diagnóstico participativo donde se involucran ambas partes (socios comunitarios y estudiantes); se planifican las etapas del proyecto estableciéndose el cronograma de intervenciones; se realiza la intervención como tal y finalmente se cierra el proceso con la elaboración del proyecto, donde deben plasmarse los productos finales.

Durante este proceso se dan, como instancias obligatorias, las tutorías por parte del docente de cátedra y las supervisiones por parte del docente de terreno. El objetivo de las tutorías es generar una conversación con los grupos de estudiantes respecto de sus vivencias con los socios comunitarios.

\section{Metodología}

De lo expuesto, se presenta una investigación de tipo documental descriptiva que proporciona elementos básicos para el diseño de una Metodología de Aprendizaje en Servicio como recurso instruccional en el desarrollo de proyectos de intervención social. A continuación, se analizan los aspectos mencionados considerados en el diseño y aplicación de la metodología de aprendizaje en servicio la cual, en el Programa de Formación Fundamental, contempla las siguientes etapas:

1. Descripción general del socio comunitario y/o destinatario, el cual se realiza con el objeto de contextualizar el trabajo a desarrollar para rea- 
lizar el proyecto de servicio solidario; se determinan las principales características de tipo demográfico, social y cultural del socio comunitario, así como el acceso a las redes y recursos.

2. Diagnóstico Social Participativo, definido como una etapa donde se establecen la naturaleza y magnitud de las necesidades o los problemas que afectan a un determinado socio comunitario y/o destinatario con la finalidad de desarrollar actividades conducentes a propiciar soluciones a las mismas. Es decir, se considera como un complejo proceso donde se evidencian necesidades, problemas y su magnitud, identificando causas y consecuencias, los recursos disponibles y la comprensión del contexto donde se desenvuelve. Se determinan las características demográficas, sociales, culturales y el acceso a redes y recursos.

Para el diagnóstico, se utilizan principalmente fuentes primarias de información, mediante la observación de los participantes de la actividad y de aquellas personas que componen el equipo de trabajo que se desempeña cotidianamente en el lugar, con el fin de percibir y clasificar a los beneficiarios y así generar un conocimiento amplio, objetivo, confiable y fehaciente acerca de los problemas que pudieran presentarse. Además, se utilizan técnicas de educación popular, para entrar en confianza con los usuarios, teniendo en consideración una dinámica que pueda aplicarse a todos, inclusive aquellos que cuentan con un nivel educacional crítico (lluvia de ideas).

3. Identificación de problemas, donde se ubican las necesidades de los involucrados de acuerdo con el área temática a ser abordada por cada especialidad (carrera); en esta etapa se jerarquizan y se fundamenta la problemática a ser abordada con la intervención (árbol del problema)

4. En base a lo anterior, se realiza la propuesta de servicio a realizarse la cual contiene objetivo general y específicos, productos y resultados esperados lo que a su vez contempla la planificación de actividades a realizarse.

5. Posteriormente se evalúan, el logro de cada uno de los objetivos específicos del proyecto a través de indicadores de logro considerando la calidad y efectividad de las actividades a realizar. 
6. Se elabora el presupuesto, el cual permite estimar en coherencia con los objetivos planteados para la ejecución del proyecto, estimando en forma clara los recursos que se necesitan y los gastos que implica.

7. Finalmente, se elaboran las conclusiones donde se reflexiona sobre la intervención y cursos de acción que se han tomado para el éxito del proceso.

En la siguiente figura se observan como se aplican las etapas de IAP en la Metodología de Aprendizaje en Servicio.

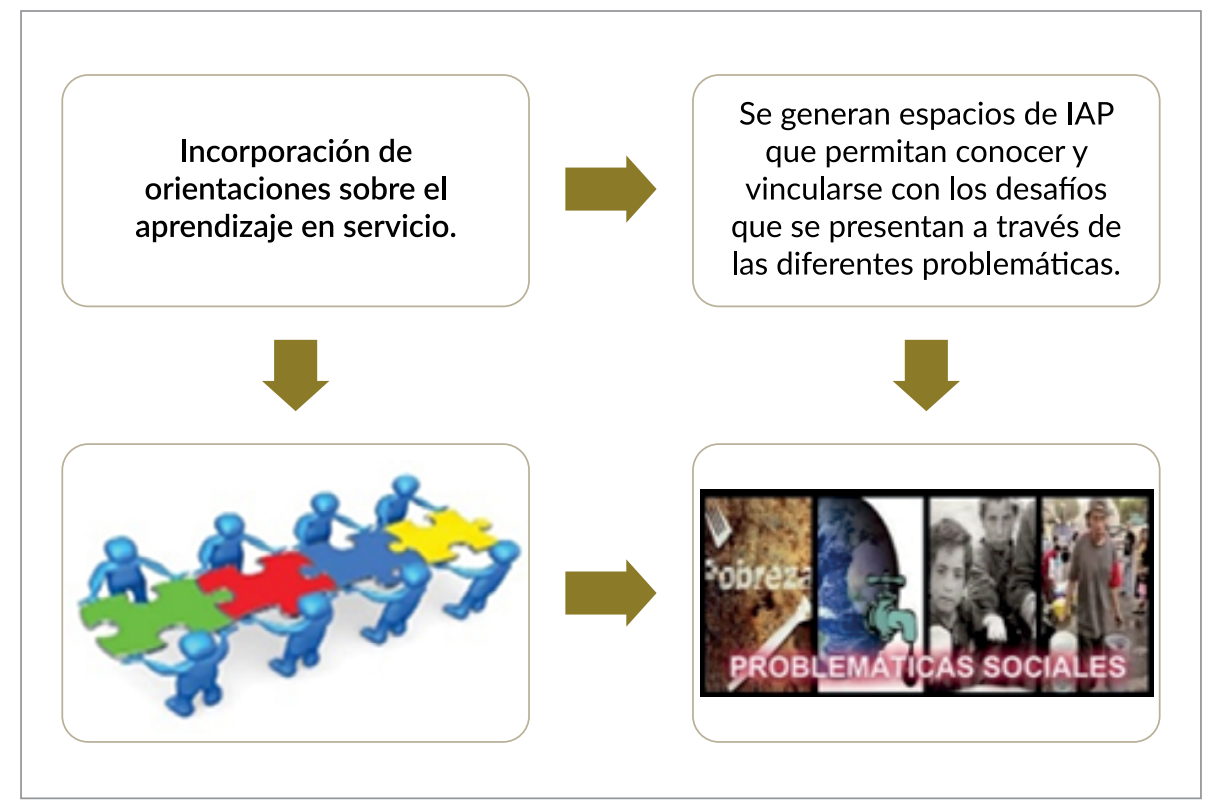

Figura 2. Investigación-acción y Aprendizaje en Servicio.

El procedimiento de intervención se realizó, durante el año 2018, de la siguiente manera:

\section{Etapa 1: Mapeo y selección de Socios Comunitarios (SC)}

En esta etapa los docentes identificaron, dentro de un margen territorial, el conjunto de Socios Comunitarios (SC) con los cuales se buscaba establecer la relación cooperativa en el marco del módulo. En el caso de SC antiguos, se analizó la factibilidad de continuar la relación colaborativa, considerando el 
cumplimiento de los compromisos de los actores involucrados y la pertinencia de los proyectos, siendo esta última definida por los directores de escuela y las mallas curriculares de cada carrera. Respecto a los nuevos SC, se buscó que la selección fuera en base al cumplimiento de los requerimientos mínimos para postular y que apuntaran a mejorar las condiciones de vida de las personas en los ámbitos de salud, educación, habitabilidad, cultura y trabajo, entre otros.

Asimismo, para la selección se consideraron temáticas emergentes relativas a inclusión social: género, diversidad sexual, derechos humanos, envejecimiento, discapacidad, migración, interculturalidad, infancia y adolescencia, pobreza y marginalidad. Se relevóla importancia de excluir como candidato a SC a cualquier institución u organización que persiguiera fines de lucro, a excepción de personas naturales, agrupaciones o cooperativas que estuvieran iniciando o fortaleciendo un emprendimiento o idea de negocio con el objeto de lograr superar condiciones de vulneración y/o pobreza.

\section{Etapa 2: Aproximación y primeras conversaciones con los SC}

Con los socios comunitarios antiguos se retomaron las conversaciones a través de llamadas telefónicas o correo electrónico, donde declararon el interés de continuar la relación cooperativa, intencionándose además una reunión presencial con el objeto de informar el funcionamiento de la línea académica durante el 2018 y sus correspondientes implicancias para ambos actores. Con los nuevos se inició una primera conversación a través de correos electrónicos

y llamadas telefónicas, donde expresaron el interés de iniciar una relación de trabajo cooperativo, describiéndose brevemente la línea académica.

\section{Etapa 3: Vinculación con el SC}

Esta etapa implicó que, mediante un contexto de reunión presencial, los docentes expusieran brevemente las características, implicancias, compromisos, productos esperados y plazos relativos a la línea académica, así como también, el beneficiario profundizó en su propósito, ámbito de acción y proyectos actuales. Por otra parte, se definieron aspectos logísticos como:

Vías de comunicación formales (celular, correo electrónico, teléfono fijo). 
Datos de contacto de los participantes, particularmente la contraparte directa y los representantes de los destinatarios u otros involucrados relevantes.

Formalización de la relación cooperativa, donde ambas partes firmaron un convenio en resguardo de lo pautado. Este documento, fue enviado vía correo para que el SC lo revisara, lo completara con los datos correspondientes, lo firmara y lo entregara a los docentes para que estos también lo hicieran, quedando ambos con una copia.

\section{Etapa 4: Prediagnóstico}

En una segunda reunión intencionada en la etapa anterior, se inició la recopilación de información relevante dando cuenta de alguna necesidad, problemática, o aspecto que se requería fortalecer (diagnóstico preliminar), evaluándose las problemáticas en virtud de los criterios de pertinencia, viabilidad, tiempo y competencia de los alumnos. Asimismo, en esta etapa los actores involucrados lograron clarificar sus expectativas, declarando que esperaba cada uno del otro, definiendo los acuerdos y compromisos que se adoptarían para el desarrollo del plan de acción del proyecto.

Además, en esta etapa se hizo entrega al SC de la copia del convenio firmado por ambas partes. Cabe señalar que las etapas 3 y 4 pudieron realizarse en una misma reunión, considerando las condiciones brindadas por el SC. Finalmente, en este lapso se consiguió que el estudiante utilizara su capacidad de comprometerse, de escuchar y dialogar, de saber mirar a través de los ojos del otro, de aprender a ponerse en su lugar, de desarrollar un pensamiento crítico, capaz de identificar las partes de un todo, su interdependencia y de entender el sentido auténtico del servicio.

Por otra parte, esta metodología se apoya en la Investigación-acción participativa (IAP) que, de acuerdo a lo planteado por Folgueiras \& Sabariego (2018), es "un método de estudio y acción que busca obtener resultados fiables y útiles para mejorar situaciones colectivas, basándose en la participación de los propios colectivos a investigar, que así pasan de ser objeto de estudio a ser sujeto protagonista" (p. 5) 
De acuerdo con las mencionadas autoras, algunas características son:

Tiene como objetivo transformar la realidad.

Comprende la realidad social como una totalidad concreta y compleja a la vez.

El proceso se plantea como una vía de movilización de los grupos sociales siendo, a la vez, un proceso sistemático de recogida de información.

Parte de las demandas o necesidades sentidas por las personas afectadas, las cuales aparecen espontáneamente o después de una primera etapa de reflexión uniendo reflexión y acción (Ibíd, p. 8)

Y los ejes centrales son:

Delimitación de objetivos que hay que trabajar conforme a la detección de determinados síntomas.

Elaboración de un diagnóstico participativo.

Puesta en práctica de acciones que se derivan del diagnóstico con su respectiva evaluación y valoración hacia un nuevo ciclo en el que se identificarán nuevos síntomas y necesidades concretándose nuevos objetivos.

En este contexto vemos como la IAP se considera como un método de investigación fundamentado en un elemento clave: la participación de distintos agentes. Se basa en la reflexión y una serie de prácticas inclusivas dirigidas a todos los integrantes de una comunidad en la creación de conocimiento científico sobre sí mismos. Es también una forma de intervenir en los problemas sociales; buscando que la misma sirva para la transformación social y está centrada en la intervención de quienes conforman la comunidad donde se interviene, ya que es la encargada de definir y dirigir sus propias necesidades, conflictos y soluciones.

Al aplicar la metodología de aprendizaje en servicio y considerando lo planteado por Mendía (2016), el servicio solidario se presta con la finalidad de atender necesidades reales de una comunidad; en este sentido, en la Universidad de Talca a través de la línea Responsabilidad Social del Programa de Formación Fundamental se desarrollaron, para el año 2018, más de 48 proyectos de innovación social con características de sustentabilidad, agrupados como sigue: 
Cuadro 1

Proyectos de Responsabilidad Social 2018.

\section{PROYECTOS DE RESPONSABILIDAD SOCIAL}

Apoyo en Plan de Negocios a microempresarios/ agricultores

Asesoría para postular a fondos de emprendimiento a microempresarios/agricultores

Desarrollo de plan de negocios a microempresarios/ Ing. Comercial agricultores

Análisis de Costos para el plan de negocios de microempresarios/agricultores

Alfabetización digital para microempresarios/ agricultores

\begin{tabular}{|c|c|}
\hline Desarrollo de identidad corporativa y branding & Diseño \\
\hline Diseño e innovación de productos & Diseño \\
\hline Diseño de medios digitales & Diseño \\
\hline Diseño de packing y puntos de venta & Diseño \\
\hline Diseño y evaluación de puestos de trabajo & Diseño \\
\hline Rediseño de espacios públicos & Arquitectura \\
\hline $\begin{array}{l}\text { Diseño de espacios para comercialización de } \\
\text { microempresarios }\end{array}$ & Arquitectura \\
\hline $\begin{array}{l}\text { Promoción de estilos de vida saludables en niños y } \\
\text { adultos mayores }\end{array}$ & Tecnología Médica \\
\hline Promoción y prevención en salud & Tecnología Médica \\
\hline $\begin{array}{l}\text { Promoción y educación en salud e higiene bucal en } \\
\text { niños y adultos mayores }\end{array}$ & Odontología \\
\hline Reminiscencia en adultos mayores & Fonoaudiología \\
\hline $\begin{array}{l}\text { Promoción de narrativa en niños por medio de } \\
\text { cuentos infantiles }\end{array}$ & Fonoaudiología \\
\hline Deglución (dirigido a cuidadores) & Fonoaudiología \\
\hline $\begin{array}{l}\text { Calidad, higiene y seguridad para } \\
\text { microempresarios/agricultores }\end{array}$ & Nutrición y Dietética \\
\hline Cursos de primeros auxilios & Enfermería \\
\hline
\end{tabular}

Ing. Comercial

Ing. Comercial Auditoría y Control de Gestión

Ing. Informática Empresarial

Diseño

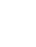

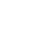


PROYECTOS DE RESPONSABILIDAD SOCIAL

CARRERA

Promoción y prevención en salud

Desarrollo de habilidades sociales

Promoción de la sana convivencia

Lenguaje musical en establecimientos educacionales

Asesoría en cuidado y mantención de áreas verdes

Promoción del cuidado del medio ambiente en establecimientos educacionales

\begin{tabular}{|c|c|}
\hline Promoción del Derecho del Trabajo & Derecho \\
\hline Promoción de Derecho Penal Adolescente & Derecho \\
\hline Promoción de Derecho de Familia & Derecho \\
\hline $\begin{array}{l}\text { Promoción de Derechos Fundamentales y sus } \\
\text { garantías }\end{array}$ & Derecho \\
\hline $\begin{array}{l}\text { Promoción en Derecho Cívico y Formación } \\
\text { Ciudadana }\end{array}$ & Derecho \\
\hline $\begin{array}{l}\text { Promoción en condiciones funcionales del adulto } \\
\text { mayor }\end{array}$ & Kinesiología \\
\hline Prevención en caídas del adulto mayor & Kinesiología \\
\hline Promoción de estilos de vida saludable & Medicina \\
\hline Educación en afectividad y sexualidad & Medicina \\
\hline Prevención del consumo de alcohol y drogas & Medicina \\
\hline Cursos de primeros auxilios & Medicina \\
\hline Promoción y prevención en salud & Medicina \\
\hline Apoyo a pequeña agricultura & Agronomía \\
\hline Optimización y captación del agua & Agronomía \\
\hline Cuidado y mantención de invernaderos & Agronomía \\
\hline Talleres Tics & $\begin{array}{l}\text { Ing. Civil en } \\
\text { Bioinformática }\end{array}$ \\
\hline Diseño de medios digitales & $\begin{array}{l}\text { Ing. Civil en } \\
\text { Bioinformática }\end{array}$ \\
\hline Minería Urbana & Ing. Civil en Minas \\
\hline
\end{tabular}

Enfermería

Psicología

Psicología

Música

Ing. Forestal

Ing. Forestal

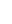




\section{Resultados de la Investigación}

Al aplicarse la Metodología de Aprendizaje en Servicio (MAS) en las diferentes carreras de la UTALCA, se obtuvieron los siguientes resultados referidos al número de proyectos por áreas:

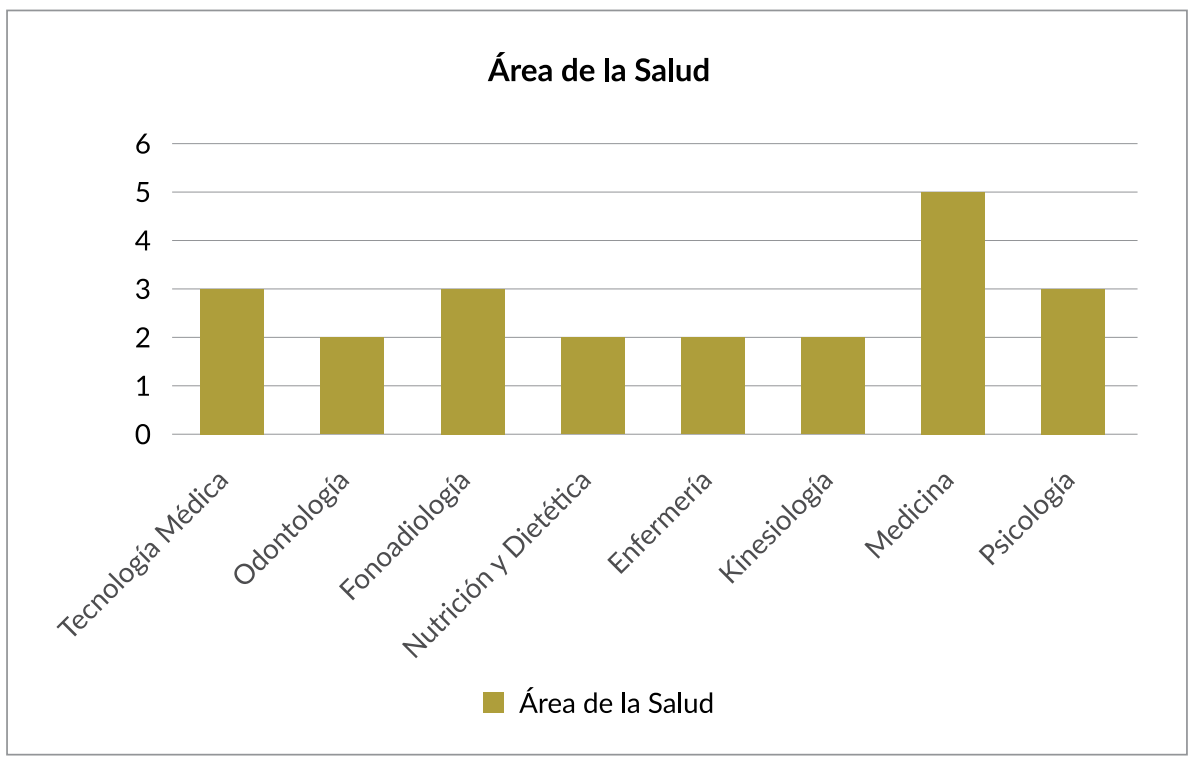

Gráfico 1. Proyectos realizados área de la salud año 2018.

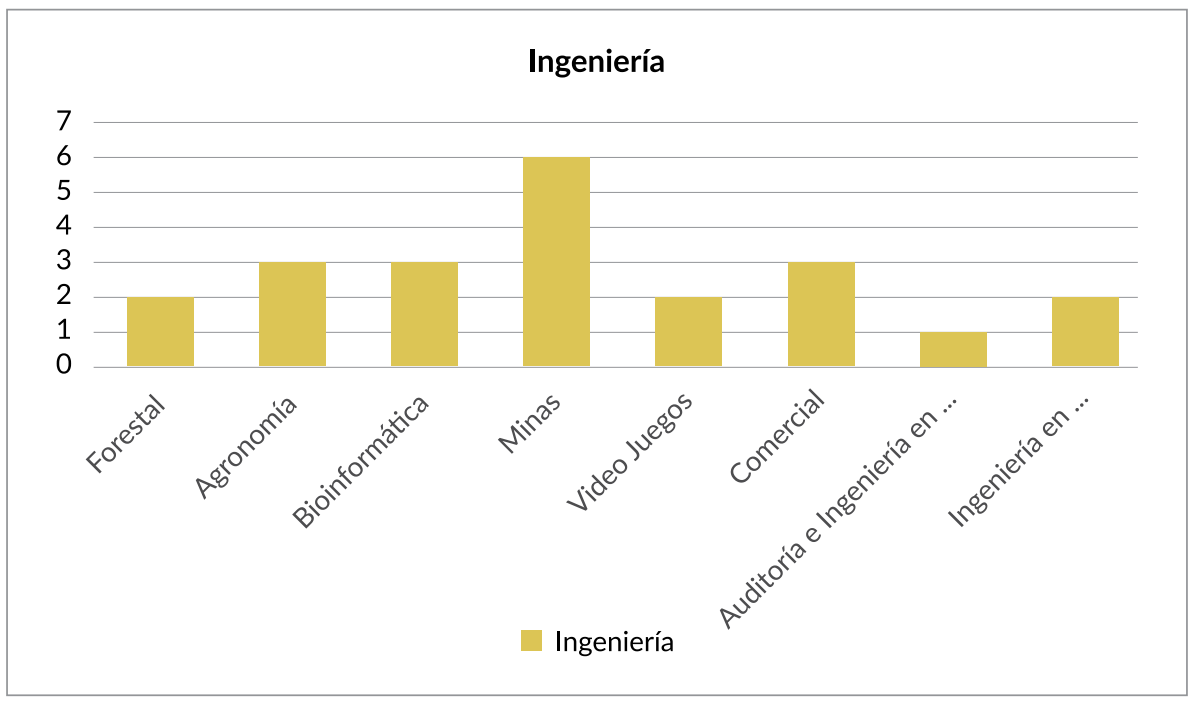

Gráfico 2. Proyectos realizados área de ingeniería año 2018. 


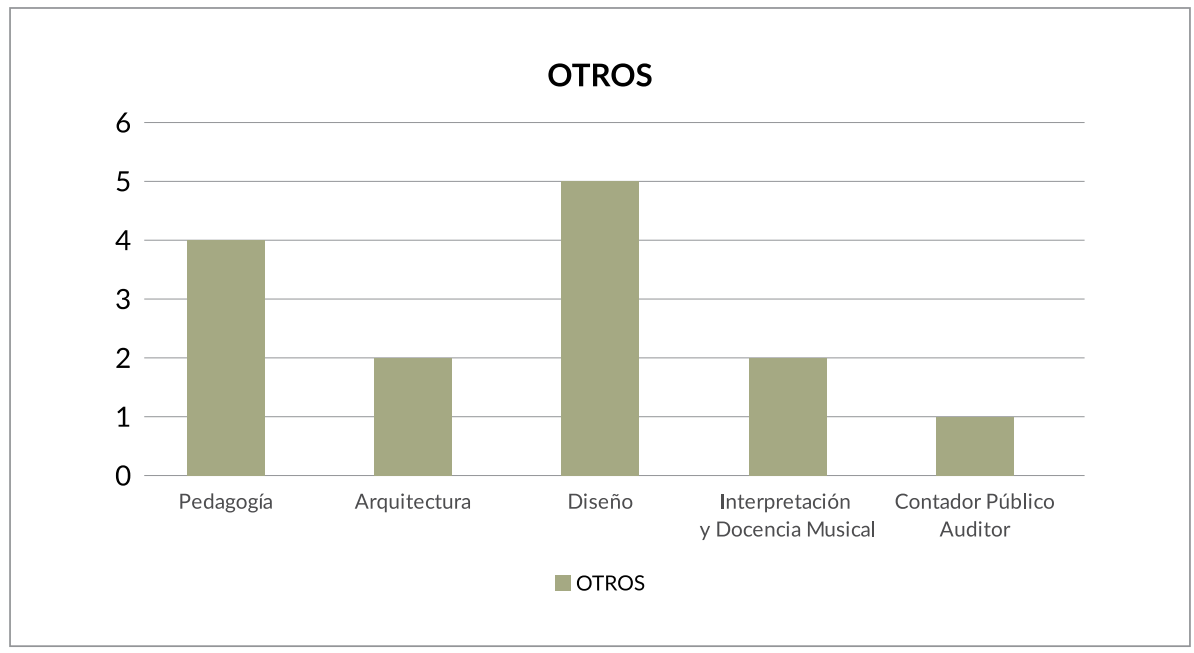

Gráfico 3. Proyectos realizados otras áreas año 2018.

Estos gráficos evidencian que al aplicarse la Metodología de Aprendizaje en Servicio se desarrollaron proyectos de intervención social. En el área de Salud se desarrollaron 22 proyectos; en el campo de las ingenierías también se desarrollaron 22 proyectos; en Pedagogía 4, en Arquitectura 2, en Diseño 5 , en Interpretación y docencia musical 2 y, finalmente en Contador público auditor 1. Todo esto totalizó para este año 58 proyectos de intervención social.

Por otra parte, se caracteriza el tipo de SC intervenido y donde se aplica la Metodología de Aprendizaje en Servicio durante el noveno semestre de carrera a través del ramo de Responsabilidad Social, evidenciándose que un mayor porcentaje de la intervención se realiza con socios comunitarios pertenecientes al sector público integrado por Casas de Adulto Mayor, centros culturales, organismos de Estado como Gendarmería y organizaciones civiles entre otros, contabilizándose 47 socios comunitarios. Esto se observa en el siguiente gráfico: 


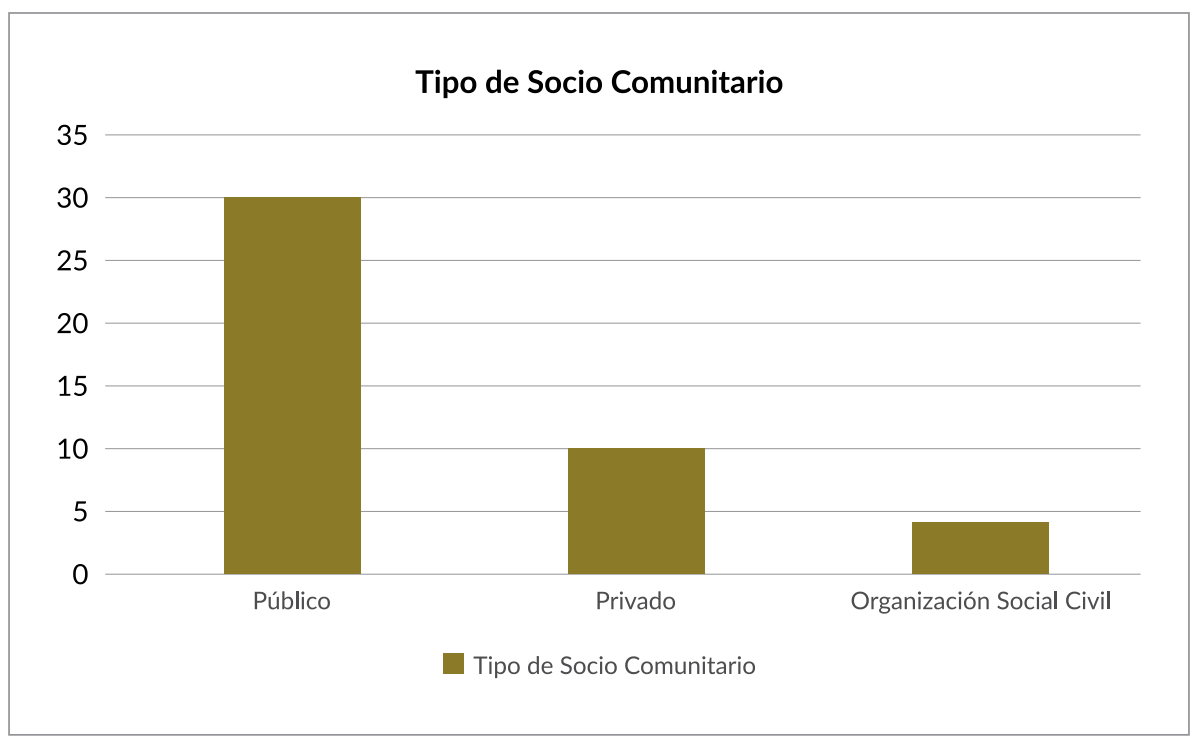

Gráfico 4. Tipos de Socios Comunitarios.

Por otra parte, el diagnóstico realizado permitió conocer y caracterizar los 47 SC que estaban activos a la fecha para desarrollar proyectos de innovación social y que estaban necesitados de una intervención por parte de los estudiantes de las diferentes carreras de la UTALCA para interactuar con éstos, desarrollando actividades educativas con componentes estéticos lúdicos de acuerdo con el perfil requerido para el desarrollo del proyecto. 
Cuadro 2

Socios comunitarios activos 2018.

\begin{tabular}{|c|c|c|c|}
\hline 1 & Club de Adulto Mayor Nazaret & 25 & Escuela La Florida \\
\hline 2 & $\begin{array}{l}\text { Club de Adulto Mayor Atardecer de } \\
\text { la Vida }\end{array}$ & 26 & Escuela Los Geranios \\
\hline 3 & Club de Adulto Mayor Los Huertos & 27 & Liceo Héctor Pérez Biott \\
\hline 4 & Club de Adulto Mayor Siempre Viva & 28 & Liceo Industrial Superior Talca \\
\hline 5 & $\begin{array}{l}\text { Corporación Cultural Barrial Brilla } \\
\text { El Sol }\end{array}$ & 29 & Liceo Artístico del Maule \\
\hline 6 & Centro Ecológico Alegría Verde & 30 & Centro Integral de Educación Talca (CIET) \\
\hline 7 & $\begin{array}{l}\text { Centro Ecológico Sinfonía de } \\
\text { Semilla }\end{array}$ & 31 & Colegio Especial Crecer \\
\hline 8 & $\begin{array}{l}\text { Centro de Capacitación de Ciegos y } \\
\text { Limitados Visuales (CENCACYLIV) }\end{array}$ & 32 & Centro de Capacitación Laboral Iberia \\
\hline 9 & $\begin{array}{l}\text { Centro Cultural de Integración } \\
\text { y Desarrollo de Discapacidad } \\
\text { (CECUDI) }\end{array}$ & 33 & Taller Laboral Unpade Talaun \\
\hline 10 & $\begin{array}{l}\text { Centro Cultural Artístico y Desarrollo } \\
\text { Social Espacio Down }\end{array}$ & 34 & $\begin{array}{l}\text { Escuela Especial Evangélica } \\
\text { Presbiteriana }\end{array}$ \\
\hline 11 & $\begin{array}{l}\text { Centro de Trastorno del Espectro } \\
\text { Autista Talca (TEA-Talca) }\end{array}$ & 35 & Corporación Abate Molina \\
\hline 12 & Jardín y Sala Cuna Manitos de Amor & 36 & Gendarmería \\
\hline 13 & $\begin{array}{l}\text { Jardín y Sala Cuna De La Cuna al } \\
\text { Mundo }\end{array}$ & 37 & Hospital Regional de Talca \\
\hline 14 & Escuela de Lenguaje Caracola & 38 & $\begin{array}{l}\text { Servicio Larga Estadía Hospital de } \\
\text { Talca }\end{array}$ \\
\hline 15 & Jardín Infantil Pelusita & 39 & $\begin{array}{l}\text { Ministerio de Vivienda y Urbanismo } \\
\text { (MINVU) }\end{array}$ \\
\hline 16 & Jardín Infantil Rapuncel & 40 & $\begin{array}{l}\text { Programa Escuelas Saludables para el } \\
\text { Aprendizaje Espacio Talca }\end{array}$ \\
\hline 17 & Centro Educacional Iris & 41 & Telecentro Sor Teresa \\
\hline 18 & Escuela Ema Escajadio & 42 & $\begin{array}{l}\text { Telecentro Comunitario Padre } \\
\text { Hurtado }\end{array}$ \\
\hline 19 & Escuela Brilla El Sol Felipe Cubillos & 43 & $\begin{array}{l}\text { Corporación Nacional Forestal } \\
\text { (CONAF) }\end{array}$ \\
\hline 20 & Escuela San Miguel & 44 & $\begin{array}{l}\text { Programa de Desarrollo Local } \\
\text { (PRODESAL) }\end{array}$ \\
\hline 21 & Escuela Villa La Paz & 45 & Instituto Nacional de la Juventud \\
\hline 22 & Escuela Básica Talca & 46 & $\begin{array}{l}\text { Servicio Nacional de la Mujer y la } \\
\text { Equidad de Género (SERNAMEG) }\end{array}$ \\
\hline 23 & Escuela Juan Luis Sanfuentes & 47 & $\begin{array}{l}\text { Servicio Nacional del Adulto Mayor } \\
\text { (SENAMA) }\end{array}$ \\
\hline 24 & Escuela Carlos Spano Talca & & \\
\hline
\end{tabular}




\section{Discusión de los resultados}

Se presentan los resultados de una manera generalizada tomando la línea de Responsabilidad Social como una unidad académica dentro del Programa de Formación Fundamental. Esto significa que para su análisis se engloban e interrelacionan todos los elementos que constituyen la Metodología de Aprendizaje en Servicio (MAS) para describirla. Desde este punto de vista están en estrecha relación con los elementos didácticos y pedagógicos donde se concibe la responsabilidad social como un medio para el aprendizaje y aplicación de las metodologías participativas. Así, este análisis contempla un entrejido de resultados relativos al contenido programático, las actividades interactivas y las estrategias de enseñanza-aprendizaje determinando, a su vez, que la aplicación de esta metodología permite la utilización de técnicas de intervención social en el modelo de educación por competencias de la universidad y la vinculación con las otras líneas del Programa de Formación Fundamental en consonancia con las competencias alcanzadas en comunicación y aprendizaje transpersonal. Estos resultados permitieron definir las áreas temáticas a ser abordadas:

Asesoría a Microempresarios y Agricultores, consistente en la promoción de información en proyectos de negocios emergentes y/o establecidos.

Promoción de la Ciencia y Tecnología, a través de talleres y otras actividades que impliquen el desarrollo de conocimientos asociados a Tecnologías de Información y de las comunicaciones (Tics).

Promoción de Derechos Ciudadanos, a través de proyectos de vinculación y formación socio educativa, brindando asesorías en áreas temáticas referidas a Derecho del Trabajo, Seguridad Social, Derecho Penal Adolescente, Derecho de Familia, Formación Ciudadana, Derechos Civiles en el área de Inclusión y de violencia.

Desarrollo de ambientes socialmente responsables, brindando asesorías para el diseño de espacios públicos.

Mejoramiento de la calidad de vida, promoviendo actividades socio educativas y realizando talleres con actividades de estimulación cognitiva en el adulto mayor, hábitos alimenticios, deglución y promoción de estilos de vida saludables. 
Educación Medio ambiental, promoviendo proyectos dirigidos a informar y apoyar a la pequeña agricultura, cuidado y mantención de invernaderos y de áreas verdes.

\section{Conclusiones}

Los escenarios y las metodologías de la enseñanza en la UTALCA experimentan una profunda renovación frente a los posicionamientos didácticos clásicos centrados en el aula y en la actividad del profesor. es decir, hoy en día se aboga por una enseñanza centrada sobre la actividad autónoma del alumno, lo que conlleva que tanto la planificación como la realización de los procesos de enseñanza-aprendizaje se lleven a cabo asumiendo este punto de vista.

En este orden de ideas, el aprendizaje en servicio proporciona innumerables contextos en los cuales aplicar los aprendizajes de competencias. Ofrece la oportunidad para desarrollar el pensamiento estratégico, no en base a simulaciones o supuestos, sino a partir de un escenario real que incluye todos los componentes: análisis de la realidad, creación, planificación, desarrollo y evaluación de un proyecto. Además, es adaptable a las circunstancias de cada grupo de estudiantes, en cualquier nivel o modalidad.

Los proyectos socio comunitarios que se realizan siguiendo esta metodología de aprendizaje en servicio están dirigidos, en su mayoría, al abordaje de problemáticas sociales en instituciones públicas y en el ámbito de acción de la universidad, ya que la responsabilidad social compromete el desarrollo de los objetivos de esta y su compromiso con el Estado; no obstante, se atienden a través de esta metodología algunos socios comunitarios de carácter privado y organizaciones civiles.

Al aplicarse la metodología de aprendizaje en servicio sustentada en la investigación acción participativa se consigue, a través del proyecto, que después de la reflexión se aporte al socio comunitario respectivo alternativas de solución innovadoras a la problemática detectada en el diagnóstico y se le proporcione un producto de responsabilidad social con características de sustentabilidad e innovación social.

La metodología de aprendizaje en servicio desarrolla en el alumno las competencias de saber, saber hacer y saber ser, ya que debe aplicar los conocimientos adquiridos durante su formación personal en un proyecto de intervención social con características de responsabilidad social. 
En el diseño de esta metodología uno de los aspectos más importantes ha sido correlacionar las necesidades de aprendizaje del estudiante con los objetivos, los contenidos programáticos y su implementación. Así pues, su contribución es decisiva ya que se generan constantes oportunidades para el aprendizaje de metodologías participativas, las cuales están diseñadas para facilitar su participación en las diversas actividades, generando constantes oportunidades para el aprendizaje adquiriendo conciencia de sus capacidades, motivándose a involucrarse activamente en el proceso de enseñanza - aprendizaje.

\section{REFERENCIAS BIBLIOGRÁFICAS}

Castillo, J. (2012). Sociología de la Educación. Estado de México: Red Tercer Milenio.

Folgueiras, P., \& Sabariego, M. (2018). Investigación-acción participativa. El diseño de un diagnóstico participativo. REIRE Revista d'Innovació i Recerca en Educació, 17(1), 16-25. Doi: http://doi.org/10.1344/reire2018.11.119047

Mendía, R. (2016). El aprendizaje-servicio: una metodología para la innovación educativa. CONVIVES, 16, 20-26. Recuperado de http://convivesenlaescuela.blogspot.com/2016/12/revista-convives-n-16-aprendizaje. html

Quezada, I. (2014). El modelo de educación por competencias y su impacto en la planificación estratégica de la Universidad de Talca (Chile). Revista Universitaria Ruta, 16(1), 7 - 18. Recuperado de https://revistas.userena.cl/index.php/ruta/article/view/466

Tobón, S. (2013). Formación Integral y Competencias. Pensamiento complejo, currículo, didáctica y evaluación. Bogotá, Colombia: Editorial ECOE.

Universidad de Talca (2018). Plan Estratégico. Desafíos, iniciativas y acciones clave. Recuperado de http://planificacion.utalca.cl/pagina/img/documento/Avance_Plan_Estrategico_2020_a2018.pdf

Universidad de Talca (2018). Syllabus: Segundo Semestre Responsabilidad Social 2018. Recuperado de http://dcc.utalca.cl/icc/Syllabi_2019/s7_ p16_EticaYRRSS.pdf 